Check for updates

Cite this: RSC Adv., 2019, 9, 3740

\title{
A Col I and BCP ceramic bi-layer scaffold implant promotes regeneration in osteochondral defects
}

\author{
Hanxu Cai, ${ }^{a}$ Ya Yao, ${ }^{a}$ Yang Xu, ${ }^{a}$ Qing Wang, ${ }^{a}$ Wen Zou, ${ }^{\text {ab }}$ Jie Liang, ${ }^{\text {*ab }}$ Yong Sun, (D) *a \\ Changchun Zhou, ${ }^{a}$ Yujiang Fan (D) and Xingdong Zhang ${ }^{a}$
}

Osteochondral defects occur in the superficial cartilage region, intermediate calcified cartilage, and subchondral bone. Due to the limited regenerative capacity and complex zonal structure, it is critically difficult to develop strategies for osteochondral defect repair that could meet clinical requirements. In this study, type I collagen (Col I) and BCP ceramics were used to fabricate a new bi-layer scaffold for regeneration in osteochondral defects. The in vitro studies showed that the bi-layer scaffold provided special functions for cell migration, proliferation and secretion due to the layered scaffold structure. The in vivo results demonstrated that the bi-layered scaffold could effectively promote the regeneration of both the cartilage and the subchondral bone, and the newly formed cartilage layer, with a similar structure and thickness to the natural cartilaginous layer, could seamlessly integrate with the surrounding natural cartilage and regenerate an interface layer to mimic the native osteochondral structure.

Received 6th November 2018

Accepted 21st December 2018

DOI: 10.1039/c8ra09171d

rsc.li/rsc-advances
To date, clinical treatments such as microfracturing, chondral shaving, abrasion arthroplasty, mosaicplasty, subchondral drilling, and prosthetic joint replacement have been available for osteochondral defect repair., ${ }^{\mathbf{1 , 4}}$ However, these therapeutic approaches still face challenges because of their limitations in therapeutic effect, such as ill-fitted donor tissue availability, donor site lesions, implant loosening, and limited durability of prosthetics. $^{7,8}$ Osteochondral defects seriously influence the patients' life and health, and they have attracted increasing attention because of the great potential cost of clinical application requirements. In the past few years, the combination of biomaterials and tissue engineering technologies has progressively become the optimal alternative for repairing complex osteochondral defects. Because cartilage and subchondral bone have different functions and properties, it is difficult to use a single type of scaffold with homogenous properties to simultaneously regenerate the two parts. ${ }^{\mathbf{1} 9}$ Developing a difunctional bilayer scaffold with two discrete layers of differing physical and chemical properties is expected to more effectively simultaneously regenerate the defective cartilage and subchondral bone.

Recently, bi-layer osteochondral scaffolds have been reported, most of which have been fabricated as an integrated scaffold in vitro and then implanted in vivo. ${ }^{\mathbf{1 0 , 1 1}}$ Usually, the obvious inadequacies and drawbacks of this method lie in the insufficient integration between the cartilage layer of the new implant and the surrounding normal cartilage tissue. In this study, the application of injectable hydrogels provided a new idea for solving this problem. The characteristic of in situ gelation overcame the challenge of graft-to-native cartilage integration and made the implant materials sufficiently match
${ }^{a}$ National Engineering Research Center for Biomaterials, Sichuan University, 29 Wangjiang Road, Chengdu 610064, P. R. China

${ }^{b}$ Sichuan Testing Center for Biomaterials and Medical Devices, Sichuan University, 29 Wangjiang Road, Chengdu, 610064, China. E-mail: sunyong8702@scu.edu.cn; jie_L88@126.com 
the shape of the defect and well-integrated with the surrounding normal tissue. Furthermore, in this study, we used a $3 \mathrm{D}$ cell culture model, which provided a better physiologic microenvironment for cells than the $2 \mathrm{D}$ model. ${ }^{12}$ Moreover, to ensure a long-lasting integration between chondral and bony layers, only using chemical or physical bonding of the biomaterials might be not the best solution, especially if the degradation of the materials does not match the efficient neoformation of extracellular matrix at the interface. Scotti et al. proposed that "biological bonding" between chondral and bony tissue by the extracellular matrix synthesized would afford an actively and durably interconnected interface even following the resorption/degradation of the implant. ${ }^{13}$ The native osteochondral interface is comprised of a calcified cartilage layer, within which hypertrophic chondrocytes are embedded and direct the mineralized matrix. ${ }^{14}$ Tortelli et al. seeded MSC onto a porous ceramic; the data indicated that MSC led to bone formation through an endochondral ossification process. ${ }^{15}$ This process relies on MSC differentiation into chondrocytes. At the periphery of ceramic pores, chondrocyte hypertrophy produced cartilaginous and mineralized matrix. ${ }^{\mathbf{1 6}}$ In our study, we expected that with the combined the effects of collagen and ceramics, the cells at the interface layer could differentiate into hypertrophic chondrocytes and the proposed biological interface layer synthesized by the extracellular matrix.

Nowadays, calcium phosphate (CaP) ceramics, such as hydroxyapatite (HA), tricalcium phosphate (TCP) and biphasic calcium phosphate (BCP), are widely used in repairing bone defects, based on their similar composition with the inorganic components of natural bone. ${ }^{17}$ Many researchers have found that $\mathrm{CaP}$ ceramic could induce bone formation in the skin or muscle tissue without the addition of other growth factors. ${ }^{\mathbf{1 8 - 2 0}}$ Previously, our lab had investigated the osteoinductive ability of $\mathrm{CaP}$ ceramics. The results showed that BCP had the strongest osteoinductive ability among HA, TCP and BCP bioceramics. ${ }^{21-23}$ According to the above results, BCP ceramics were selected as the subchondral bone repair layer. In articular cartilage, collagen is the main component of the extracellular matrix. Collagenous matrices provide a lot of biological functions to maintain cell and growth factor transport into the cartilage lesion. ${ }^{24}$ Thus, it is important to develop an adequate collagen framework in cartilage repair. Although the cartilage matrix mainly contains type II collagen (Col II), Rutgers et al. reported that no significant differences between Col I and II were observed in cartilage repair, and no hypertrophic phenomenon was confirmed. ${ }^{25}$ Furthermore, previous research in our research group showed that Col I hydrogel might induce chondrogenic differentiation of mesenchymal stem cells (MSCs), and the induction was material-dependent. ${ }^{26}$ Therefore, we chose Col I hydrogel to repair the cartilage lesion.

We fabricated a novel bi-layered scaffold designed to mimic the native osteochondral grafts by the construction of three distinct but seamlessly integrated layers: Col I hydrogel as cartilage layer, BCP ceramic as subchondral bone, and an interface region consisting of Col I hydrogel and BCP ceramic. Firstly, BCP ceramic was implanted as subchondral bone layer to provide a suitable mechanical environment for cartilage regeneration and promote the repair of subchondral bone. Subsequently, the neutral Col I solution with encapsulated exogenous chondrocytes was injected on the surface of the BCP ceramic and rapidly gelatinized to mimic the characteristics of natural extracellular matrices and serve as cartilage layer. Then, the viability, morphology, proliferation and metabolic activity of chondrocytes in the scaffold were assessed in vitro. After that, we transplanted the bi-layer scaffold into the osteochondral defect, and the regeneration ability in the osteochondral defect was assessed by macroscopic assessment and staining results. We expected the bi-layer scaffold could effectively regenerate tissues in the osteochondral defect.

\section{Materials and methods}

\subsection{Materials}

Fresh biphasic calcium phosphate ceramic (BCP, HA : $\beta$-TCP $=$ $2: 8, \phi 10 \times 2 \mathrm{~mm}, \phi 4 \times 3 \mathrm{~mm}$ ) and type I collagen (Col I, extracted from calf skin) were provided by National Engineering Research Center in Biomaterials, Sichuan University. Phosphate-buffered saline (PBS) and $\alpha$-minimum essential medium $(\alpha$-MEM) were purchased from Thermo Fisher Scientific Corporation (USA). Fetal bovine serum (FBS, Gibco, Australia origin) was purchased from Life Technologies Corporation (USA). Ascorbic acid (Vc) was purchased from Sigma-Aldrich.

\subsection{Bi-layered scaffold fabrication and characterization}

Bi-layered osteochondral scaffold was fabricated via two steps. Firstly, BCP ceramic was placed in a mold $(10 \mathrm{~mm}$ in diameter and $5 \mathrm{~mm}$ in length) as the subchondral bone layer and soaked in PBS. Then, the $\mathrm{pH}$ value of the Col I solution was regulated to 7.2 by addition of $1.0 \mathrm{M} \mathrm{NaOH}$ in ice-bath while PBS was added to keep the final concentration of Col I at $10 \mathrm{mg} \mathrm{mL}^{-1}$. The neutral Col I solution (about $0.24 \mathrm{~mL}$ ) was poured into the mold at $37{ }^{\circ} \mathrm{C}$ for $0.5 \mathrm{~h}$. The Col I could gel quickly on the surface of the calcium phosphate ceramic as cartilage layer to form the bilayered osteochondral scaffold. The scaffold was frozen in liquid nitrogen and immediately lyophilized. After that, crosssections of the scaffolds were coated with a layer of gold to observe the morphology and interior structure by scanning electron microscopy (SEM, HITACHI S-800, Japan).

The equilibrium swelling ratio played an important role in maintaining the structural stability of materials. It also could test whether the bi-layer scaffold cracked and separated under physiological conditions. The bi-layered scaffold was prepared using the method described above. In addition, the monolayer collagen scaffold was used as the control group. Then, the bilayered scaffold and single Col I hydrogel were immersed into $10 \mathrm{~mL}$ phosphate buffer ( $\mathrm{pH} 7.4$ ) and placed into a shaker in a $37{ }^{\circ} \mathrm{C}$ air bath and shaken at $90 \mathrm{rpm}$ (ZHWY-2012C, Shanghai Zhicheng, China). At the appointed time interval, the residual moisture of the two kinds of scaffolds was removed by filter paper, and the weight and diameter of each sample were measured three times. At the same time, samples were observed 
for whether the bi-layer scaffolds cracked and separated under physiological conditions.

\subsection{Isolation and culture of rabbit chondrocytes}

Articular cartilage specimens were isolated from the femurs and tibias of New Zealand white rabbits that were three to five days old. Articular cartilage was carefully extracted and cleaned well in PBS with $10 \%$ penicillin-streptomycin. Thereafter, articular cartilage specimens were cut into pieces, followed by treatment with $0.25 \mathrm{mg} \mathrm{mL}^{-1}$ trypsin for 30 minutes, and then with $1 \mathrm{mg}$ $\mathrm{mL}^{-1}$ of collagenase type II for 6 hours at $37^{\circ} \mathrm{C}$. The obtained cell suspension was filtered through a $70 \mu \mathrm{m}$ nylon cell strainer (BD) and centrifuged at $1200 \mathrm{rpm}$ for 5 minutes.

The isolated chondrocytes were cultured in $\alpha$-MEM at $37^{\circ} \mathrm{C}$ under $5 \% \mathrm{CO}_{2}$ containing $10 \%$ fetal bovine serum, $1 \%$ penicillin-streptomycin, and $0.01 \%$ phosphate-Vc.

\subsection{Cell viability, morphology and quantification of GAG content}

BCP ceramics were sterilized by $\gamma$-ray irradiation to avoid altering the physiochemical properties of materials before use. The $\mathrm{pH}$ value of the Col I solution was regulated to 7.2 by addition of $1.0 \mathrm{M} \mathrm{NaOH}$ in ice bath, and PBS was added to keep the concentration of the collagen solution at $10 \mathrm{mg} \mathrm{mL}^{-1}$. Then, the chondrocyte suspension was mixed with the collagen solution $\left(5 \times 10^{6}\right.$ cells $\left.\mathrm{mL}^{-1}\right)$. After that, collagen solution with encapsulated chondrocytes was poured on the surface of the ceramics and placed at $37{ }^{\circ} \mathrm{C}$ for $0.5 \mathrm{~h}$. Lastly, the bi-layered constructs with encapsulated chondrocytes were detached from the mold and immersed into $\alpha$-MEM containing $10 \%$ FBS, $0.01 \%$ phosphate-Vc and $1 \%$ penicillin-streptomycin, and incubated at $37^{\circ} \mathrm{C}$ under $5 \% \mathrm{CO}_{2}$.

After 3 and 7 days of culture, the viability of cells was observed by FDA/PI (live/dead) staining using confocal laser scanning microscope (CLSM, Leica TCSSP5, Germany), and the morphology of chondrocytes was observed using SEM.

The chondrogenic matrix glycosaminoglycan (GAG) was quantified after 3 and 7 days of culture, respectively. Briefly, both the type I collagen hydrogel and bi-layered scaffold with encapsulated chondrocytes were flash-frozen in liquid nitrogen and freeze-dried. The lyophilized samples were digested in a $0.1 \%$ papain solution at $60{ }^{\circ} \mathrm{C}$ for 12 hours. The supernatants were collected and tested for DNA and GAG content. The DNA content was measured by Hoechst 33258 (B1302, Sigma). The GAG content was measured by a Blyscan sGAG assay kit (B100, Biocolor). Each test was performed with at least three experimental samples in parallel, and the results are presented as the ratio of GAG to DNA.

\subsection{Bi-layered scaffold for repairing osteochondral defects in rabbit}

2.5.1 Animal surgery. All animal studies were approved by the Sichuan University Medical Ethics Committee. All animal procedures were performed in accordance with the guidelines for care and use of laboratory animals of Sichuan University.
12 mature New Zealand White rabbits (about 2.5-3 kg) were purchased from Chengdu Dashuo Laboratory Animal Co. Ltd. and cared for by Sichuan Testing Center for Biomaterials and Medical Devices. After intravenous injection of pentobarbital sodium (40 $\mathrm{mg} \mathrm{kg}^{-1}$ ), the knee joint was opened using a medial parapatellar approach, and the patella was dislocated laterally. Then, an osteochondral defect (4 mm diameter, $4 \mathrm{~mm}$ depth) was constructed on the trochlear ridge with a stainless drill. This is larger than the critical-size defect for cartilage self-healing. ${ }^{27}$ Each animal received bilateral surgery. Then, the osteochondral defects were randomly assigned to one of the four groups, and each group had three parallel samples: (1) empty defect, (2) single-layer scaffold (type I collagen with $5 \times 10^{6}$ cells per $\mathrm{mL}$ chondrocytes, $0.050 \mathrm{~mL}$ ), (3) single-layer scaffold (BCP ceramic $\phi 4 \times 3 \mathrm{~mm}$ ), (4) bi-layered scaffold (BCP ceramic $\phi 4 \times 3 \mathrm{~mm}$, type I collagen with $5 \times 10^{6}$ cells per $\mathrm{mL}$ chondrocytes $\phi 4 \times 1 \mathrm{~mm}, 0.013 \mathrm{~mL}$ ). In all groups, BCP ceramic was pressed into the defect sites, while the type I collagen with encapsulated chondrocytes was injected into the sites, and then was allowed to gel in situ. The joint capsule and skin were closed with interrupted sutures.

After the surgery, rabbits were immediately sent back to their individual cages to allow skin incisions to heal and were allowed full weight bearing. Then, postoperative antibiotic (gentamicin) was administered intramuscularly at a dose of $400000 \mathrm{U}$ per day for 3 days.

2.5.2 Gross morphology observation and assessment. After two and four weeks, animals were sacrificed to collect samples. Once the joints were opened, the defect sites and surrounding joint tissues were examined and photographed. Three samples of each group were blindly and independently assessed by three evaluators using the International Cartilage Repair Score (ICRS) system. ${ }^{28}$

These samples were fixed in $4 \%$ paraformaldehyde for 7 days, and subsequently decalcified in 10\% EDTA for 6 weeks. Paraffin sections $(5 \mu \mathrm{m})$ were stained with hematoxylin-eosin (HE), toluidine blue (TB) and Safranin-O (Saf.O), and counterstained with fast green to evaluate the cell morphology and GAGs distribution. Collagen II (Col II) was detected with mouse anti-rabbit collagen II (Novus Biologicals) immunohistochemical (IHC) staining. Saf.O and HE staining were used to analyze the thickness of cartilage and the content of neo-bone by Image-Pro Plus software. The new bone content was measured from the area of new bone and total area. The data were expressed as average new bone area (new bone area/total area). Both thickness and new bone content were calculated as the average of three samples in each group.

\subsection{Statistical analysis}

Statistical analysis comparing different scaffolds at different weeks was performed by one-way ANOVA followed by post hoc tests. All analyses were performed using SPSS software, and the significance level was set at $p<0.05$.

\section{Results}

\subsection{Bi-layered scaffold structure and characterization}

Bi-layered osteochondral scaffold (Fig. 1A-a) mimicked the native osteochondral structure by constructing three 
independent but continuous regions: Col I hydrogel as cartilage layer, the interface region of Col I hydrogel and BCP ceramics as intermediate transition layer, and BCP ceramics as bone region. The SEM results reveal the internal microstructure in different layers of the bi-layer scaffold (Fig. 1A-b-d). The Col I fibers were densest and uniform in diameter, they could entwine each other to form three-dimensional networks with homogeneous pore distribution and could effectively maintain the nutrient and growth factor transport, supporting cell adhesion and proliferation in the stable 3D environment. The BCP ceramic was packed with a lot of macropores and micropores, and the total porosity was above $70 \%$. The structure and phase content could significantly improve cell attachment and support vascular ingrowth and new bone formation. ${ }^{18,22}$ Furthermore, before Col I gelation, the Col I solution infiltrated into the macropores and micropores of BCP ceramic to form an intermediate transition layer (Fig. 1A-c). Because of the presence of the intermediate transition layer, bony and cartilage layer materials could seamlessly combine to form an integrated implant for osteochondral defect repair.

As shown in Fig. 1B, the macroscopic appearance and size of both Col I single-layer scaffold (Fig. 1B- $\mathrm{a}_{1}$ ) and Col I/BCP bilayered scaffold (Fig. 1B- $\mathrm{b}_{2}$ ) remained fixed after immersion in PBS for 7 days. Meanwhile, the bony and cartilage layer materials were still tightly connected, without peeling. Total quality of both Col I single-layer scaffold and Col I/BCP bi-layered scaffold also kept unchanged from day 1 to day 7 (Fig. 1C). From the above description, it is obvious that the bi-layer scaffold had good stability and could effectively maintain its structure, then further provide a stable environment for in vitro and in vivo cell growth and proliferation.

\subsection{Cell viability, morphology and quantification of GAG content}

As mentioned in previous reports, Col $\mathrm{I}$ is an ideal material for cartilage regeneration, ${ }^{24-26}$ while the porous BCP ceramic is recognized as an ideal bone repair material. ${ }^{21,22}$ Therefore, the combination of Col I and porous BCP ceramic would play an important role in osteochondral defect repair. However, what is noteworthy is whether the addition of porous BCP ceramic would influence the viability, activity and adhesion properties of chondrocytes in the cartilage layer. As shown in Fig. 2, live/dead staining and SEM were used to observe cells viability and morphology. The CLSM pictures in Fig. 2A indicate that most chondrocytes encapsulated in both the Col I hydrogel and bilayer scaffold were still alive and showed obvious cell proliferation after 3 and 7 days of culture. As presented in SEM results, after 3 and 7 days, chondrocytes adhered and spread out with a fibroblast-like morphology inside the Col I hydrogel. The results showed that both collagen and BCP ceramic could support chondrocyte adhesion, spreading and migration. Combined with the results of CLSM and SEM, results show cell viability and cell morphology had no obvious difference between the Col I hydrogel and bi-layer scaffold. This indicates that the addition of BCP ceramic did not change the biocompatibility of Col I nor affect the viability of the chondrocytes.

Fig. 2A shows the macroscopic morphology of Col I hydrogel (left) and bi-layer scaffold (right) after 3 (a) and 7 ( $\left.a_{1}\right)$ days of culture. There was a significant contraction in the Col I hydrogel group; the diameter of Col I hydrogel rapidly declined from $4 \mathrm{~mm}$ in 3 days to $2 \mathrm{~mm}$ in 7 days. In contrast, the size of bi-layer scaffold remained almost unchanged with the increase in culture time. Chondrocyte-mediated contraction of the matrices

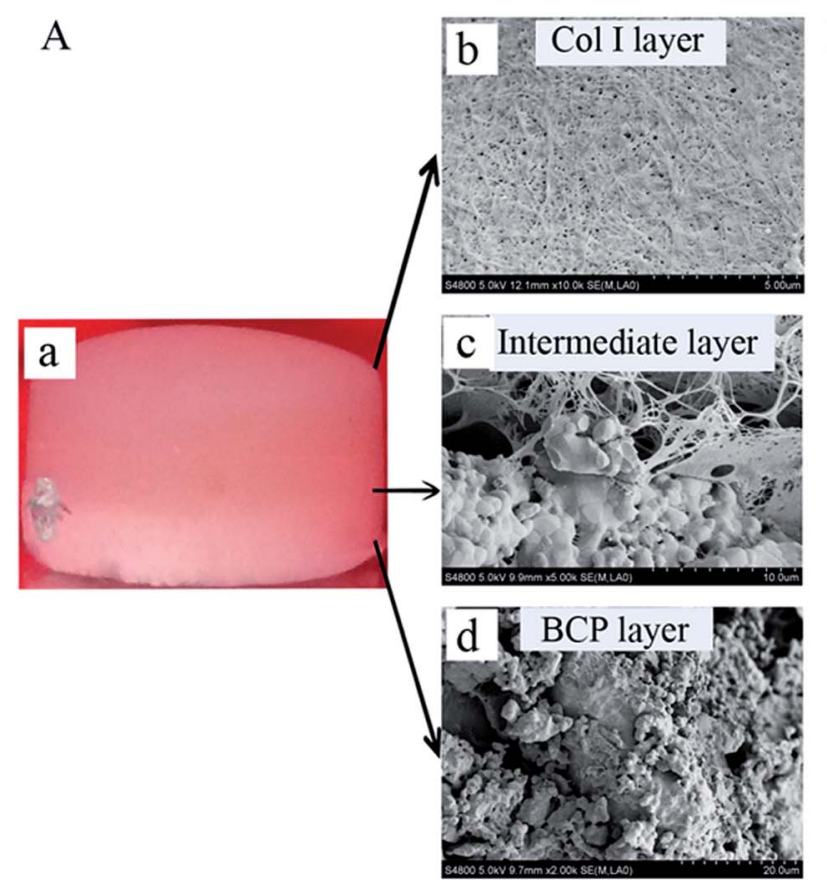

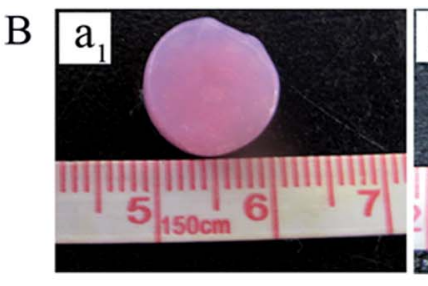

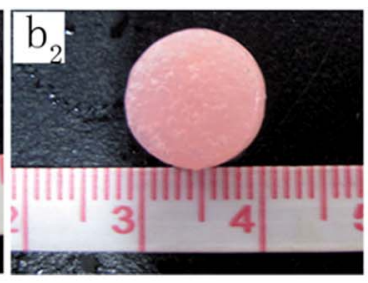

C

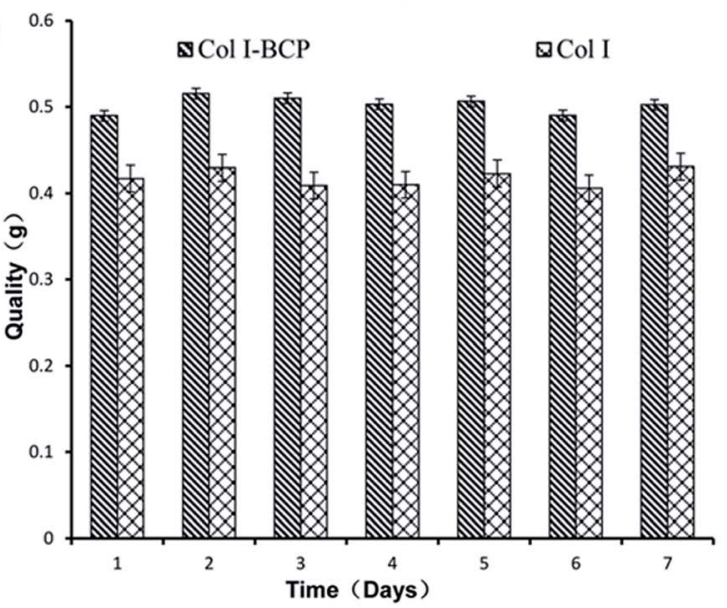

Fig. 1 (A) Structure of the bi-layer scaffolds observed by SEM. (B) Morphological changes of Col I $\left(a_{1}\right)$ and bi-layer $\left(b_{2}\right)$ scaffolds after immersion in PBS for 7 days. (C) The equilibrium swelling ratio change after immersion in PBS at $\mathrm{pH} 7.4$ and $37{ }^{\circ} \mathrm{C}$. 
A

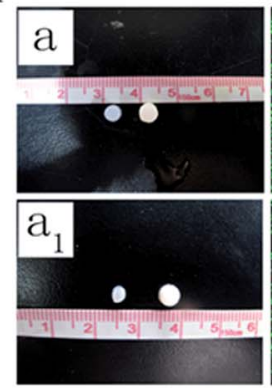

$3 \mathrm{~d}$

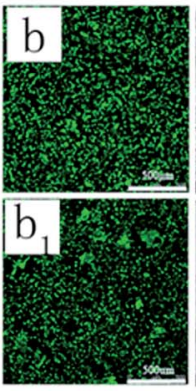

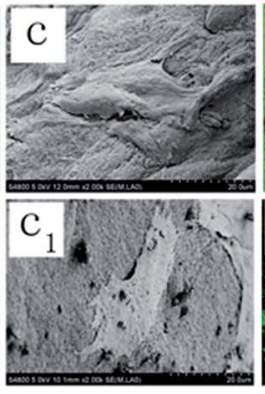

$7 \mathrm{~d}$

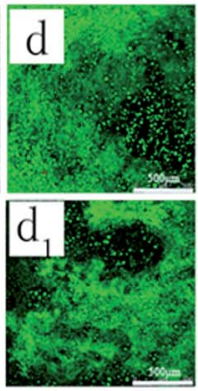

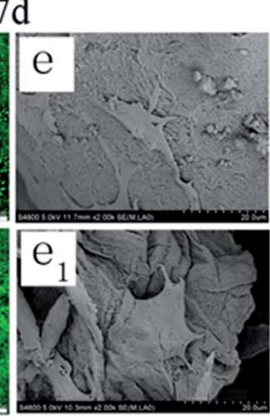

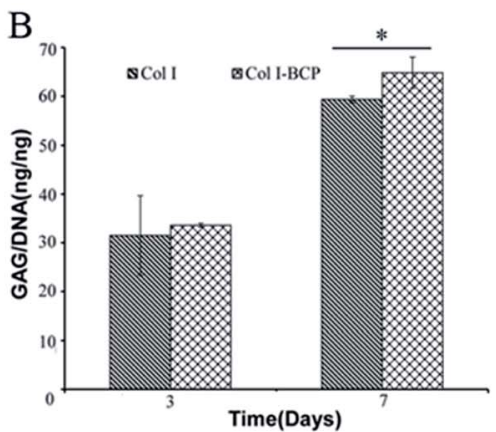

Fig. 2 The Col I hydrogel and bi-layer scaffolds with cultured chondrocytes ( $5 \times 10^{6}$ cells per $\mathrm{mL}$ ) in vitro. (A) Macroscopic morphology changes of Col I hydrogel (left) and bi-layer scaffold (right) after 3 (a) and $7\left(a_{1}\right)$ days of culture. The CLSM micrographs of chondrocytes encapsulated in Col I hydrogel after culture for 3 (b) and 7 (d) days, and the in bi-layer scaffold after culture for 3 days $\left(b_{1}\right)$ and 7 days $\left(d_{1}\right)$. The SEM micrographs of chondrocytes encapsulated in Col I hydrogel after culture for 3 days (c) and 7 days (e), and in bi-layer scaffold after culture for 3 days ( $c_{1}$ ) and 7 days $\left(e_{1}\right)$. (B) The quantification of GAG content secreted by chondrocytes after 3 and 7 days culture. * $p<0.05$.

is a serious drawback for the application of such scaffolds in articular cartilage tissue engineering. In vivo deformation of the matrix could result in a loss of contact between the implanted device and the host tissue, thereby decreasing the chances for successful integration of the repair tissue. ${ }^{29}$ Therefore, the combination of Col I and porous BCP ceramic significantly improved the ability of anti-contraction by the mechanical support of the subchondral BCP ceramic, which would facilitate the recovery of the osteochondral defect.

GAG, as a major component of the extracellular matrix, reflects the expression and function of the cells. The data from quantitative analysis of GAG (Fig. 2B) within the cartilage layer showed that the proportions of GAG to DNA in both Col I hydrogel and bi-layer scaffold increased with culture period extension, and the ability of chondrocytes to secrete GAG in the Col I/BCP bi-layer scaffold was superior to that in the Col I hydrogel mono-layer scaffold. This result indicates that the Col I/BCP bi-layer scaffold enhanced the cell proliferation ability. The probable cause was that the combined BCP ceramic prevented the Col I hydrogel from shrinking, as discussed above, and provided more cell growth space to promote chondrocyte proliferation.

\subsection{Bi-layered scaffold for repairing osteochondral defects in rabbit}

3.3.1 Macroscopic assessment of defect repair. Osteochondral defects were created on the rabbit patellar groove blank, and Col I, BCP and bi-layer scaffold were implanted as shown in Fig. 3A. After 2 or 4 weeks, the joints were opened, and synovial fluid was found to be clear and normal in all cases. There was no inflammatory response, and no scaffold delaminated or migrated from the defect into the joint cavity.

After 2 weeks (Fig. 3 Ae-h and $e_{1}-h_{1}$ ), some level of defect fill was observed in all four groups, from the cross-sectional view of the defect. The cartilage thickness of the four groups was still very thin. BCP ceramics had not degraded and also provided enough support for the cartilage layer. ICRS macroscopic scores
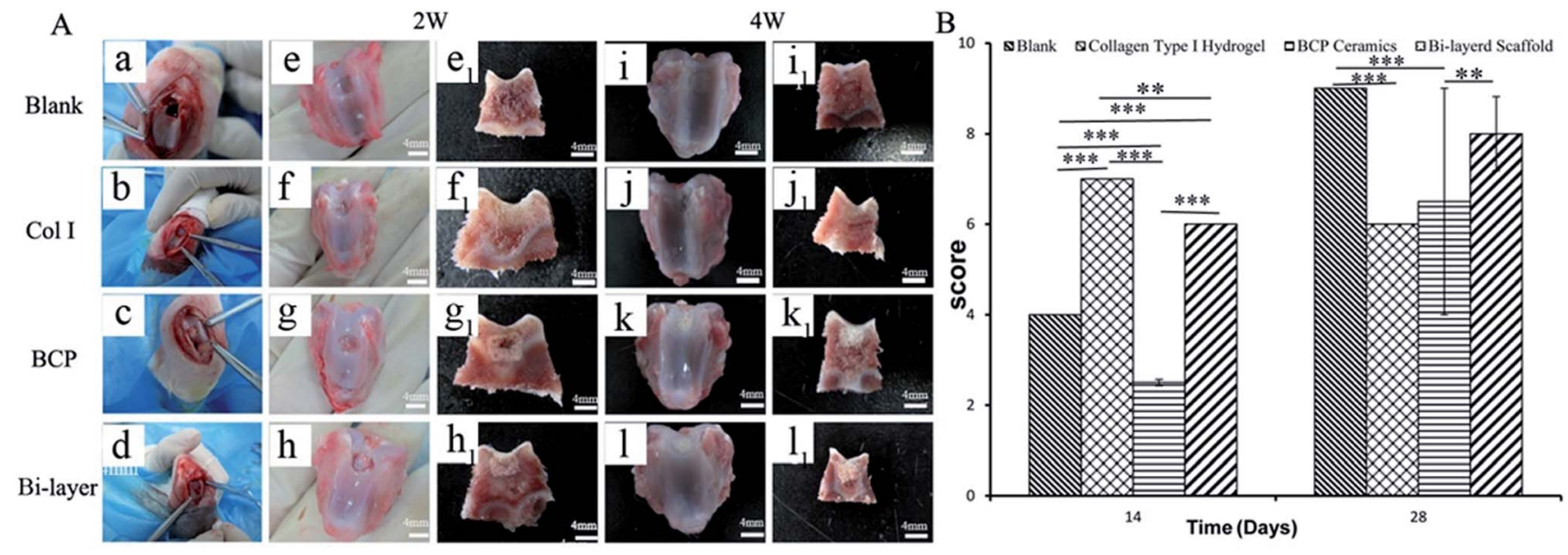

Fig. 3 Scaffolds were implanted into defects for in vivo treatment of osteochondral defects. (A) Osteochondral defects were created on the patellar trochlear groove and then filled with different scaffolds or no treatment as control (a-d). Gross and section morphology after 2 weeks $\left(\mathrm{e}-\mathrm{h}\right.$ and $\mathrm{e}_{1}-\mathrm{h}_{1}$ ) and 4 weeks $\left(\mathrm{i}-\mathrm{l}\right.$ and $\mathrm{i}_{1}-\mathrm{l}_{1}$ ). (B) Macroscopic evaluation according to ICRS macroscopic scores. ${ }^{*} p<0.05, * * p<0.01, * * * p<$ 0.001. 

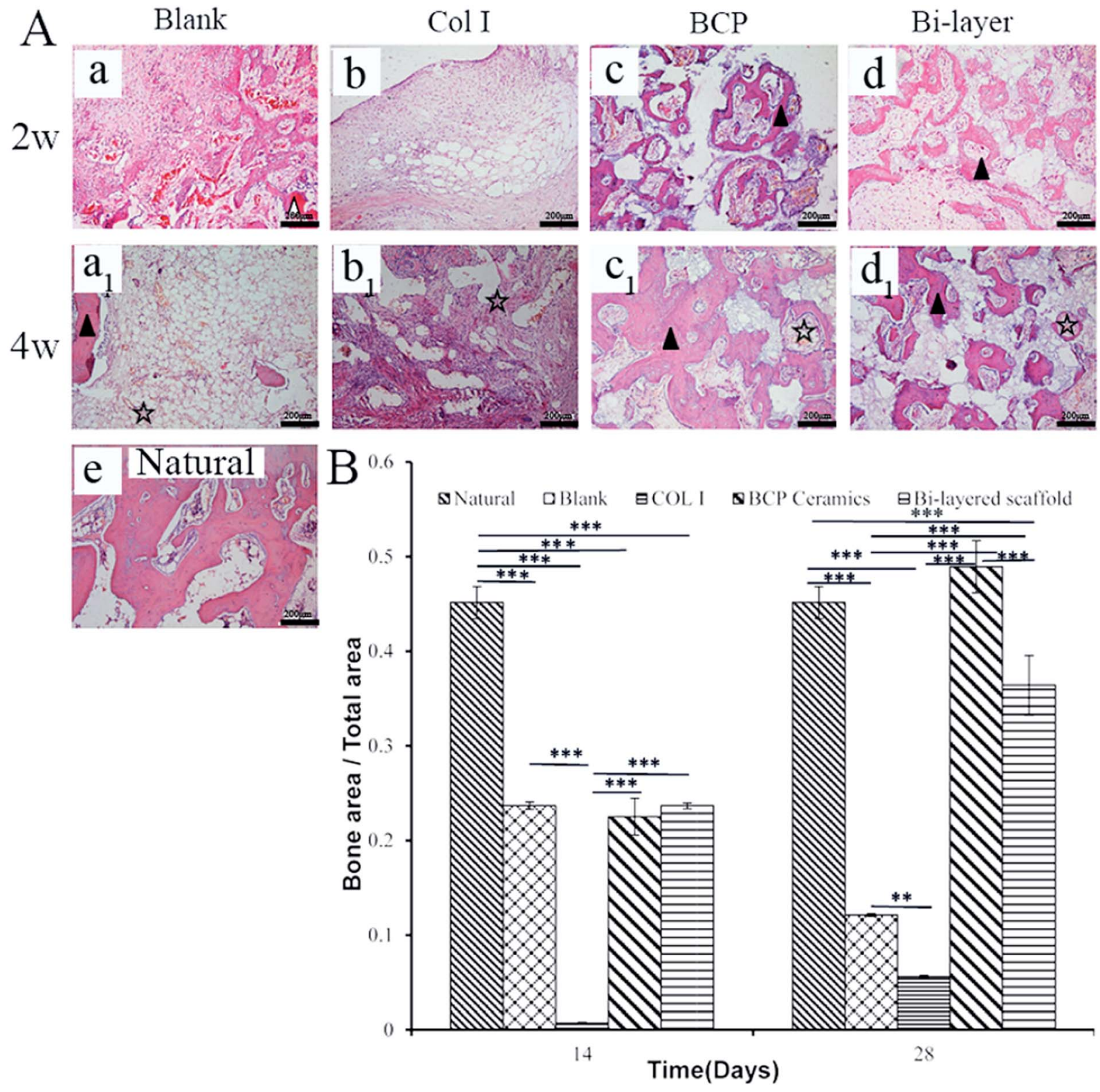

Fig. 4 (A) HE staining to analyze subchondral bone repair. (B) The density of regenerated bone at 2 and 4 weeks was calculated by Image-Pro Plus software. $\Delta-$ new bone, $\Delta$-osteoid, $\star$-blood vessel. $* p<0.05, * * p<0.01, * * * p<0.001$

revealed that the cartilage layer repair effect of the Col I group and Col I/BCP bi-layer group were better than that of the blank and BCP group (Fig. 3B).

After 4 weeks, blank, BCP ceramic and bi-layer scaffold groups nearly filled with newly formed cartilage tissue, but the Col I group was not repaired completely due to blood clots (Fig. 3Ai-l and $i_{1}-l_{1}$ ). The ICRS macroscopic scores were consistent with the findings of the visual evaluation (Fig. 3B). The blank group displayed a higher score compared to the bilayer scaffold group. This result was mainly caused by blood, which was mixed with Col I solution in the operative process before collagen gelation in some samples; subsequently, blood clot occupied the partial cartilage surface of the bi-layer and Col I scaffold and affected the final repair effect (Fig. 7e and f). These results reminded us of the importance of avoiding bleeding during operation. However, the score of the Col I/BCP bi-layer scaffold group was still higher than that of the BCP and Col I group.

3.3.2 Subchondral bone evaluation. Osteogenesis was detected in all groups by histological analysis (Fig. 4A). New bone was found in the BCP ceramic in both the BCP group and bi-layer scaffold group 2 weeks after the implantation. Furthermore, increased new bone, containing osteocytes and newly presented bone trabeculae, was discovered 4 weeks after implantation. Besides, well-developed blood vessels were observed in bone formation sites. In contrast, a small area of osteoid was observed in the blank group after 2 weeks, while there was only a small amount of new bone formation after 4 weeks. However, no bone was detected in the Col I group after 2 and 4 weeks of implantation, only fibrous tissues and blood vessels. 


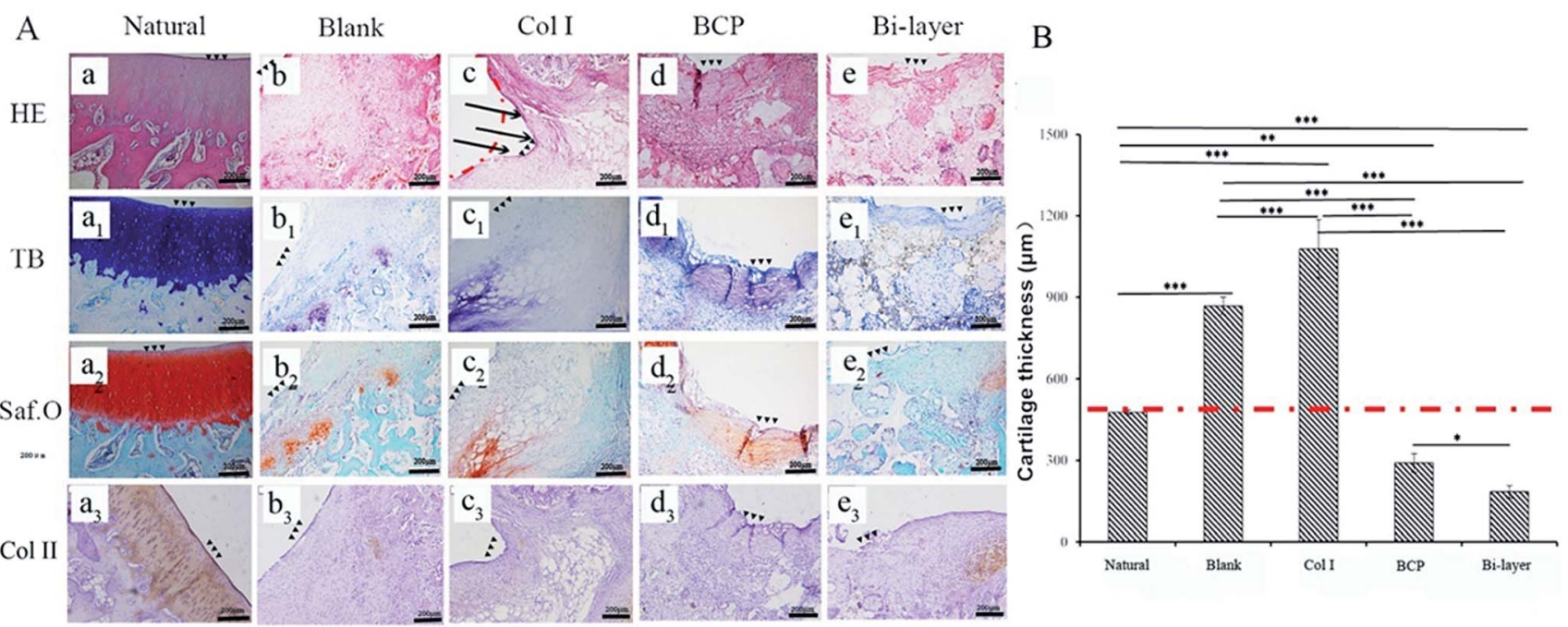

Fig. 5 (A) HE, TB and Saf.O staining images show the regeneration status of tissues after 2 weeks. (B) Cartilage thickness was calculated by Image-Pro Plus software. The dashed line and arrows indicate the collapse of cartilage due to absence of subchondral bone support. $\mathbf{\Lambda} \mathbf{\Lambda}-$ the surface of cartilage, $* p<0.05, * * p<0.01, * * * p<0.001$.

Fig. 4B shows the percentage of new bone or osteoid formation in four groups after 2 and 4 weeks of implantation. The results were consistent with HE staining. Much higher yields of new bone formed in the BCP and bi-layer scaffold groups than the blank and Col I groups. Furthermore, new bone content in the BCP group was slightly higher than that in the bi-layer scaffold group. The results might be ascribed to the Col I on the surface of BCP ceramic forming neo-cartilage rather than bone in vivo.

In a word, BCP ceramics, as the subchondral bone layer, can promote new bone formation. With the degradation of BCP ceramic material, the new bone will replace the role of ceramic as bone layer to support cartilage repair.
3.3.3 Cartilage evaluation. After 2 weeks of implantation, due to the short repair time, four experimental groups were stained weakly with GAG and Col II staining in the region of cartilage defects and even incomplete regeneration of cartilage (Fig. 5A). Compared with other groups, the Col I group showed a significant collapse due to the absence of subchondral bone support (Fig. 5A-c). The results show the importance of subchondral bone in osteochondral defect repair.

After 4 weeks, the four experiment groups displayed various degrees of hyaline-like cartilage formation, as presented by TB, Saf.O and IHC staining (Fig. 6A). The blank group displayed intense GAG and Col II staining. Some studies showed that

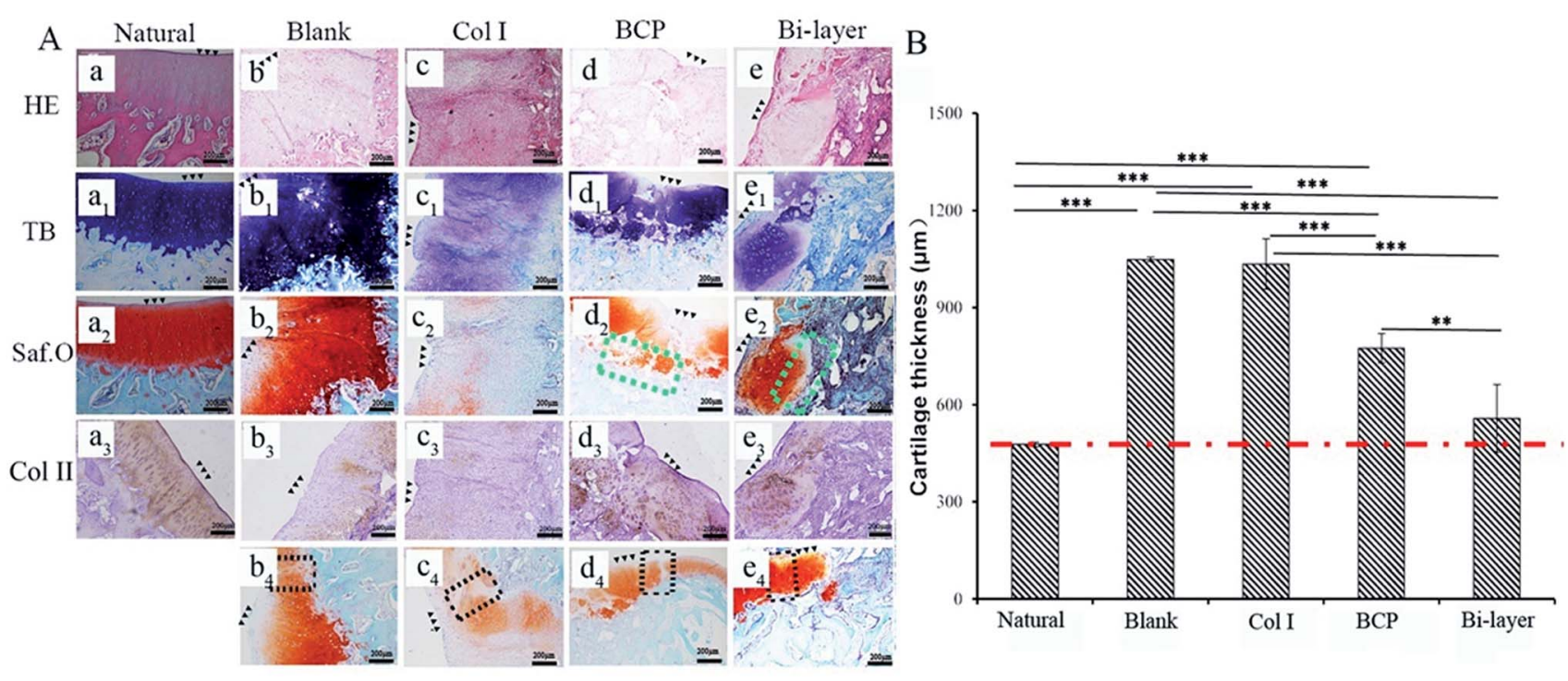

Fig. 6 (A) HE, TB and Saf.O staining images show the regeneration status of tissue after 4 weeks. (B) Cartilage thickness was calculated using Image-Pro Plus software. The green dashed boxes indicate the connection between the cartilage and the subchondral bone. The black dashed boxes indicate the connection between the newly formed cartilage tissue and surrounding healthy cartilage. $\mathbf{\Lambda} \mathbf{\Lambda} \mathbf{\Delta}-$ the surface of cartilage, $* p<$ $0.05, * * p<0.01, * * * p<0.001$. 

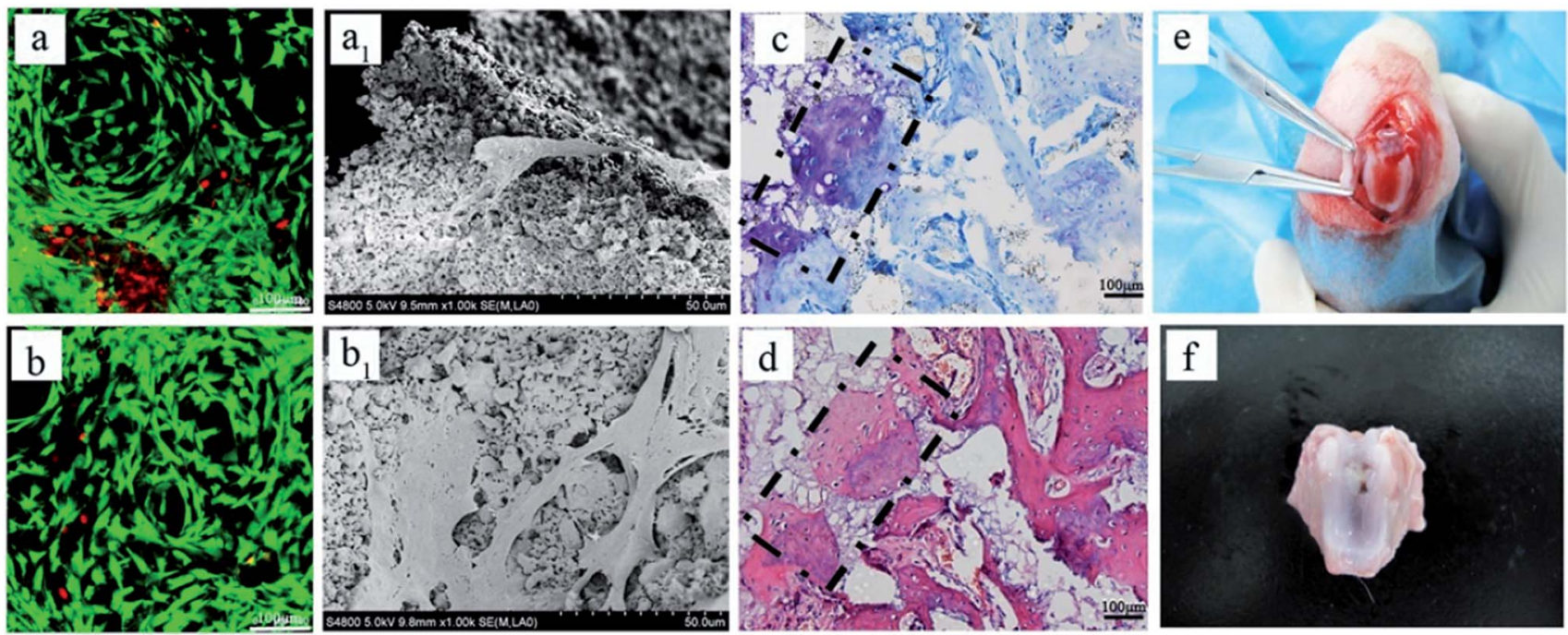

Fig. 7 The CLSM micrographs of chondrocytes encapsulated in bi-layer scaffold after culture for (a) 3 and (b) 7 days. The SEM micrographs of chondrocytes encapsulated in bi-layer scaffold after culture for $\left(a_{1}\right) 3$ and $\left(b_{1}\right) 7$ days. (c) TB and (d) HE staining images show the regeneration status of cartilage-bone interface after 4 weeks. During operation, (e) bleeding affected type I collagen gelation and (f) blood clot affected the repair effect of the cartilage layer.

during microfracture or subchondral bone drilling surgery, the subchondral bone is stimulated by drilling to expose the underlying bone mesenchymal stem cells (BMSCs) and other cells. At first, BMSCs could differentiate into chondrocytes and secrete cartilage matrix, but a large number of fibroblasts expressing type I collagen also enters, forming fibrocartilage without the mechanical robustness of hyaline tissue, and the repaired tissue was vulnerable to mechanical joint forces and typically deteriorated. ${ }^{30-32}$ Similarly, although the BCP group initially displayed intense GAG and Col II staining, the BMSCs emerge from the bone marrow with other fibroblasts, and subsequent degeneration might also occur after several months post-operation. Meanwhile, the results show no efficient interconnection between the cartilage and the bone layer, except that filled with fibrous tissue (Fig. 6A- $\mathrm{d}_{2}$ ). Hence, both therapeutic methods were not desirable in the long term.

The Col I group displayed weak GAG and Col II staining, probably due to the influence of blood coming from the operative process and lack of mechanical support, which was not conducive to cartilage layer repair, especially for load-bearing osteochondral defect repair. Therefore, this treatment might only apply to cartilage repair, but not to osteochondral defect repair.

The bi-layered scaffold displayed an intense GAG and Col II staining; the bone and cartilage layers were seamlessly connected with each other (Fig. $6 \mathrm{~A}-\mathrm{e}_{2}$ ). In this group, cells of the cartilage layer were chondrocytes rather than fibrocytes, and Col I played a role in maintaining the cartilage spherical phenotype and promoting cartilage matrix secretion. ${ }^{24-26}$ Therefore, this group is expected to maintain the long-term repair. This requires longer implantation experiments to further verify. In addition, the bi-layered scaffold group revealed that the newly formed cartilage tissue could integrate into the surrounding healthy cartilage, as indicated by staining results observation, but no conspicuous connection was found in the other three groups (Fig. $6 \mathrm{Ab}_{4}-\mathrm{d}_{4}$ ). Nevertheless, during the operation, blood in the bone marrow cavity could not be effectively inhibited, resulting in mixing with the collagen layer to form blood clots, which affected the final repair effect (Fig. 7e and f). This problem needs to be solved in the subsequent operation procedure.

As shown in Fig. 5B, the thicknesses of the new cartilage in the four groups were different from that of natural cartilage after 2 weeks of implantation. The values of empty and Col I groups were higher than that of the natural cartilage group, while those of the BCP and Col I/BCP groups were obviously lower than that of natural cartilage. However, the thickness of neo-cartilage of the bi-layer scaffold group was the nearest that of healthy cartilage than the other three groups after 4 weeks of implantation, as described in Fig. 6B.

Summarizing the above results, the Col I/BCP bi-layer scaffold was the nearest to natural cartilage tissue in morphology, thickness, and GAG and Col II expression. Besides, in this group, the bone, newly formed cartilage and native cartilage were seamlessly connected with each other. However, the blood from the bone marrow cavity seriously affected the repair effect; thus, improving the operation procedure might be an effective approach.

3.3.4 Cartilage-bone interface evaluation. Fig. 7 shows the cartilage-bone interface of the Col I/BCP bi-layer scaffold from in vitro and in vivo experiment results. The interfacial chondrocytes grew, adhered and spread around the BCP ceramic pore walls, no longer maintaining the round morphology but a fibroblast-like morphology on the surface of BCP ceramic (Fig. 7a, a1, b and b1). According to the results of HE and TB staining after 4 weeks of implantation, there was a seamless connection between the cartilage matrix and osteoid in the bilayer scaffold interface (Fig. 7c and d). The result is similar to the process of endochondral ossification as previously reported..$^{15,16,33}$ Meanwhile, it is also consistent with the postnatal bone development process, in which hypertrophic chondrocytes 
launch a process of endochondral ossification and form a thin calcified layer that arrests vascular invasion. ${ }^{\mathbf{3 4 , 3 5}}$ After a longer repair period, it is expected to form a good interface that would place the bone and cartilage in two different environments for their respective utility, and the bone and cartilage would be closely connected with each other.

\section{Conclusions}

A bi-layered osteochondral scaffold was used to simulate the native osteochondral interface by integrating Col I hydrogel and BCP ceramics. The results demonstrated that the bi-layered scaffold could effectively promote regeneration of the subchondral bone, while the newly formed cartilage layer, with a similar structure and thickness to natural cartilage, could seamlessly integrate with the surrounding natural cartilage and bone tissue, hopefully regenerating the cartilage-bone interface to mimic the native osteochondral structure and maintain longterm stable repair. This scaffold is expected to further improve on the integrated path to acquire superior osteochondral implants in future research.

\section{Conflicts of interest}

There are no conflicts to declare.

\section{Acknowledgements}

This work was sponsored by the National Key R\&D Plan (Grant No. 2018YFC1106803 and 2016YFC1103202), Sichuan Province Key Technology Research and Development Program (2016SZ0003), Young Elite Scientists Sponsorship Program by CAST (2017QNRC001), and Sichuan University Innovation Spark Project (2018SCUH0089).

\section{Notes and references}

1 S. P. Nukavarapu and D. L. Dorcemus, Biotechnol. Adv., 2013, 31, 706.

2 M. Sartori, S. Pagani, A. Ferrari, V. Costa, V. Carina,

E. Figallo, M. C. Maltarello, L. Martini, M. Fini and

G. Giavaresi, Mater. Sci. Eng., C, 2017, 70, 101.

3 P. Chen, C. Xia, J. Mo, S. Mei, X. Lin and S. Fan, Mater. Sci. Eng., C, 2018, 91, 190.

4 D. J. Hunter, Osteoarthritis Cartilage, 2009, 17, 1402.

5 S. Lu, J. Lam, J. E. Trachtenberg, E. J. Lee, H. Seyednejad, J. van den Beucken, Y. Tabata, M. E. Wong, J. A. Jansen, A. G. Mikos and F. K. Kasper, Biomaterials, 2014, 35, 8829.

6 D. B. Burr, Osteoarthritis Cartilage, 2004, 12, 20.

7 S. J. Seo, C. Mahapatra, R. K. Singh, J. C. Knowles and H. W. Kim, Tissue Eng., 2014, 5, 1-14.

8 S. C. Espinosa and J. C. White, Int. J. Pharm., 2017, 523, 476. 9 X. Duan, X. Zhu, X. Dong, J. Yang, F. Huang, S. Cen, F. Leung,

H. Fan and Z. Xiang, Mater. Sci. Eng., C, 2013, 33, 3951.

10 X. Ding, M. Zhu, B. Xu, J. Zhang, Y. Zhao, S. Ji, L. Wang, L. Wang, X. Li, D. Kong, X. Ma and Q. Yang, ACS Appl. Mater. Interfaces, 2014, 6(19), 16696.
11 T. J. Levingstone, A. Ramesh, R. T. Brady, P. A. J. Brama, C. Kearney, J. P. Gleeson and F. J. O'Brien, Biomaterials, 2016, 87, 69.

12 M. P. Lutolf and J. A. Hubbell, Nat. Biotechnol., 2005, 23, 47. 13 C. Scotti, D. Wirz, F. Wolf, D. J. Schaefer, V. Burgin, A. U. Daniels, V. Valderrabano, C. Candrian, M. Jakob, I. Martin and A. Barbero, Biomaterials, 2010, 31, 2252.

14 N. T. Khanarian, N. M. Haney, R. A. Burga and H. H. Lu, Biomaterials, 2012, 33, 5247.

15 F. Tortelli, R. Tasso, F. Loiacono and R. Cancedda, Biomaterials, 2010, 31, 242.

16 C. Scotti, B. Tonnarelli, A. Papadimitropoulos, A. Scherberich, S. Schaeren, A. Schauerte, J. Lopez-Rios, R. Zeller, A. Barbero and I. Martin, Proc. Natl. Acad. Sci. U. S. A., 2010, 107, 7251.

17 X. Chen, J. Wang, Y. Chen, H. Cai, X. Yang, X. Zhu, Y. Fan and X. Zhang, J. Mater. Chem. B, 2016, 4, 2280.

18 H. P. Yuan, C. A. Blitterswijk, K. D. Groot and J. D. Bruijn, J. Tissue Eng., 2006, 1607.

19 L. Wang, B. Zhang, C. Bao, P. Habibovic, J. Hu and X. Zhang, PLoS One, 2014, 9, 107044.

20 J. Yao, X. Li, C. Bao, C. Zhang, Z. Chen, H. Fan and X. Zhang, J. Biomater. Appl., 2010, 24, 607.

21 Z. Tang, Y. Tan, Y. Ni, J. Wang, X. Zhu, Y. Fan, X. Chen, X. Yang and X. Zhang, Mater. Sci. Eng., C, 2017, 70, 1000.

22 J. Wang, Y. Chen, X. Zhu, T. Yuan, Y. Tan, Y. Fan and X. Zhang, J. Biomed. Mater. Res., 2014, 102, 4234.

23 Y. Chen, J. Wang, X. D. Zhu, Z. R. Tang, X. Yang, Y. F. Tan, Y. J. Fan and X. D. Zhang, Acta Biomater., 2015, 11, 435.

24 T. Aigner, Adv. Drug Delivery Rev., 2003, 55, 1569.

25 M. Rutgers, D. B. Saris, L. A. Vonk, M. H. van Rijen, V. Akrum, D. Langeveld, A. van Boxtel, W. J. Dhert and L. B. Creemers, Tissue Eng., Part A, 2013, 19, 59.

26 L. Zheng, H. S. Fan, J. Sun, X. N. Chen, G. Wang, L. Zhang, Y. J. Fan and X. D. Zhang, J. Biomed. Mater. Res., 2010, 93, 783.

27 ASTM, Standard Guide for in vivo Assessment of Implantable Devices Intended to Repair or Regenerate Articular Cartilage, American Society for Testing and Materials, 2010.

28 M. P. Borne, N. J. Raijmakers, J. Vanlauwe, J. Victor, S. N. de Jong, J. Bellemans and D. B. Saris, Osteoarthritis Cartilage, 2007, 15, 1397.

29 A. D. Berendsen, L. A. Vonk, B. Zandieh-Doulabi, V. Everts and R. A. Bank, J. Tissue Eng. Regener. Med., 2012, 6, 721.

30 H. Imhof, I. Sulzbacher, S. Grampp, C. Czerny, S. Youssefzadeh and F. Kainberger, Invest. Radiol., 2000, $35,581$.

31 I. M. Khan, R. Williams and C. W. Archer, Cell Stem Cell, 2009, 4, 282.

32 E. A. Makris, A. H. Gomoll, K. N. Malizos, J. C. Hu and K. A. Athanasiou, Nat. Rev., 2015, 6, 21-34.

33 F. Yu, X. Cao, L. Zeng, Q. Zhang and X. Chen, Carbohydr. Polym., 2013, 97, 188.

34 H. M. Kronenberg, Nat. Rev., 2012, 25(2), 85-97.

35 C. Hoemann, C.-H. Lafantaisie-Favreau, V. Lascau-Coman, G. Chen and J. Guzmán-Morales, J. Knee Surg., 2012, 25, 085. 